\title{
Synchronous Primary Renal Lymphoma and Renal Cell Carcinoma: Collision Tumors in the Same Kidney
}

\author{
Neil J. Kocher, MD, Stephan Leung, BS, Nabeel E. Sarwani, MD, \\ Joshua I. Warrick, MD, and Jay D. Raman, $\mathrm{MD}^{1}$
}

\begin{abstract}
Background: Primary renal lymphoma (PRL) is an exceptionally rare disease with under 50 reported cases in the literature. PRL is an aggressive condition that can present with nonspecific symptoms and local invasion mimicking renal cell carcinoma (RCC). We present an unusual case involving a collision tumor between PRL and RCC.

Case Presentation: The patient is a 62-year-old immunosuppressed man with an incidental left renal mass on cross-sectional imaging. Renal mass biopsy confirmed clear cell type RCC. He underwent robot-assisted, laparoscopic left radical nephrectomy for presumed RCC without evidence for extrarenal disease or discernable lymphadenopathy. Final pathology revealed a collision tumor, including PRL and RCC.

Conclusion: To our knowledge, this is the first reported case within the literature describing a collision tumor between PRL and RCC. Although rare, it is important to consider PRL in the differential diagnosis of a solid renal mass, especially in patients with a prior history of transplantation and/or chronic immunosuppression.
\end{abstract}

Keywords: primary renal lymphoma, collision tumor, renal cell carcinoma, kidney cancer

\section{Introduction and Background}

$\mathbf{P}$ RIMARY RENAL LYMPHOMA (PRL) is an exceptionally rare disease with only 49 reported cases in the literature. ${ }^{1}$ PRL is defined as non-Hodgkin's lymphoma involving the kidney in the absence of additional organ or nodal involvement. PRL is an aggressive disease that can present with nonspecific symptoms and local invasion mimicking renal cell carcinoma (RCC). ${ }^{2}$ It is typically treated with systemic chemotherapy and targeted monoclonal therapy. ${ }^{3}$

In this study, we present the case of a 62-year-old immunocompromised man with an incidentally discovered left renal mass. Renal mass biopsy was performed and confirmed clear cell type RCC. Preoperative CT and MRI were negative for extrarenal disease or discernible lymphadenopathy. The patient underwent robot-assisted, laparoscopic left radical nephrectomy with final pathology revealing a collision tumor, including PRL and RCC. PRL is an important entity to include in the differential diagnosis for a solid renal mass, especially in patients with a prior history of transplantation and/or chronic immunosuppression.

\section{Presentation of Case}

A 62-year-old Caucasian man presented to his primary care physician with generalized lower abdominal pain. The patient had multiple medical conditions including coronary artery disease, poorly controlled type 2 diabetes mellitus, Charcot-Marie-Tooth neuropathy, and end-stage renal disease status after deceased donor renal transplantation currently on chronic immunosuppression. He denied flank pain, dysuria, or gross hematuria. Physical examination was unremarkable beyond a right lower quadrant transplant scar. Laboratory work, including a basic metabolic panel, complete blood count, tacrolimus level, and urinalysis with microscopy, was unremarkable. Noncontrast abdominopelvic CT scan revealed a $6 \mathrm{~cm}$ mixed solid and cystic left lower pole lesion arising from the native left kidney, as well as an adjacent $1.8 \mathrm{~cm}$ soft tissue nodule (Fig. 1). No suspicious lymphadenopathy or extrarenal disease was noted. CTguided renal mass biopsy of the $6 \mathrm{~cm}$ lesion confirmed RCC. The patient elected to undergo a robot-assisted, laparoscopic left radical nephrectomy with repair of a $2 \mathrm{~cm}$ umbilical

\footnotetext{
${ }^{1}$ Division of Urology, Department of Surgery, Penn State Health Milton S. Hershey Medical Center, Hershey, Pennsylvania.

Departments of ${ }^{2}$ Radiology, and ${ }^{3}$ Pathology, Penn State Health Milton S. Hershey Medical Center, Hershey, Pennsylvania.
} 


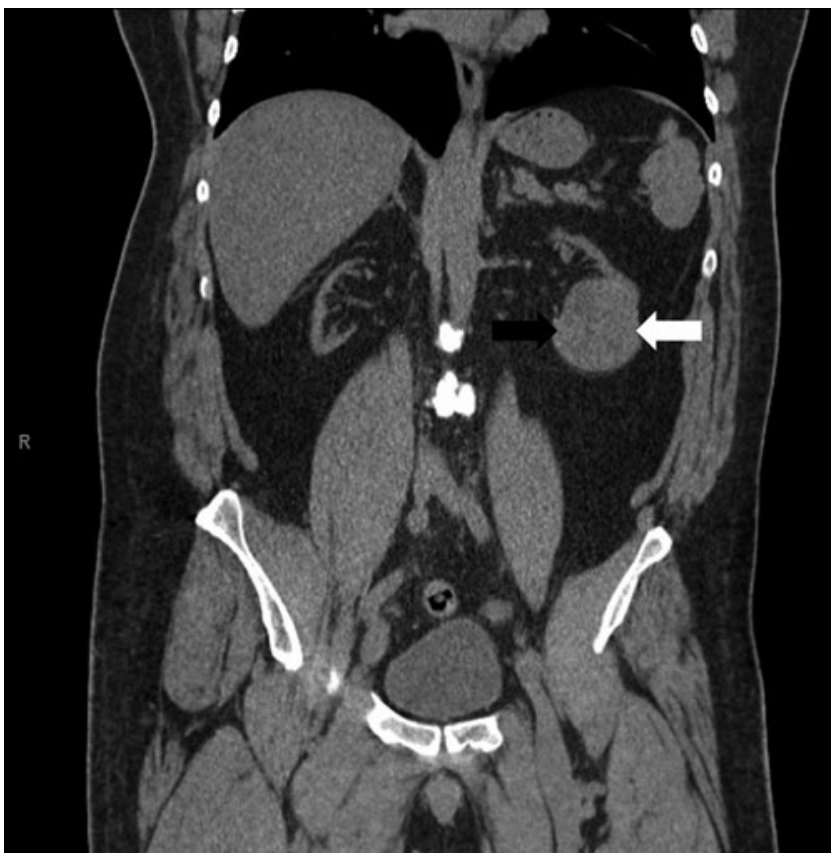

FIG. 1. Noncontrast CT coronal image. There is a $6 \mathrm{~cm}$ mixed solid and cystic lesion arising from the native left lower renal pole (white arrow) with an adjacent medial $1.8 \mathrm{~cm}$ soft tissue nodule (black arrow).

hernia. The case proceeded without complication and he was discharged home on postoperative day 2 .

Sections of the kidney mass identified a collision of two neoplasms with different morphologies (Fig. 2). The first mass measured $3.5 \mathrm{~cm}$ and was composed of nests of tumor

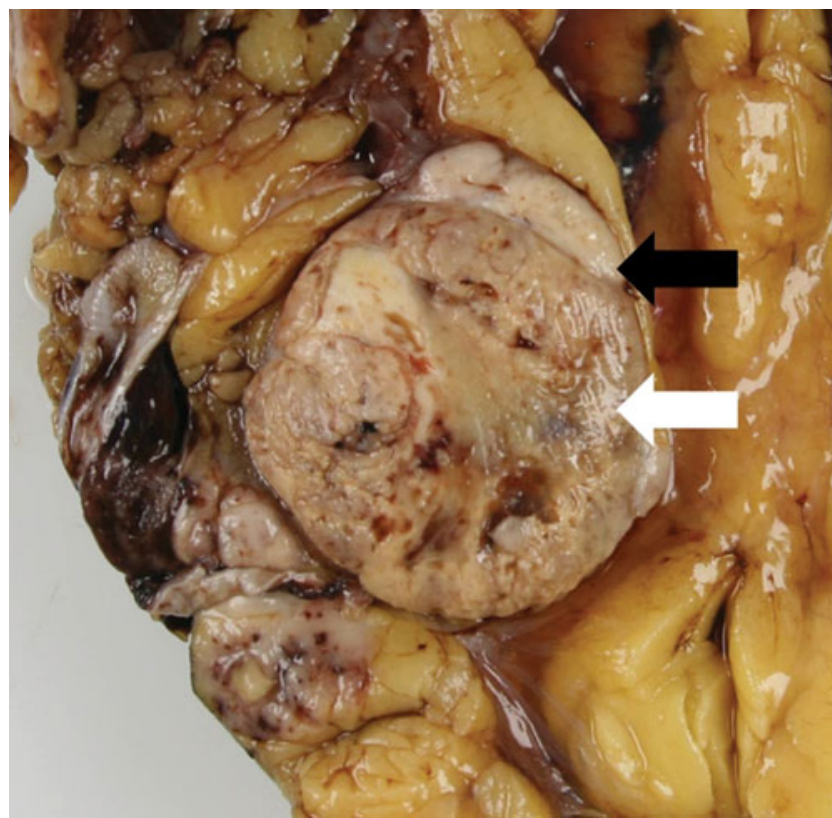

FIG. 2. Gross specimen sectioning revealed a circumscribed mass with a central scar and granular texture (white arrow). A portion of the mass was distinct with a smooth texture and white appearance (black arrow).

cells with optically clear cytoplasm, set within a delicate vascular network (Fig. 3A). Immunohistochemistry on this part showed tumor cells positive for CA-IX and PAX-8, corroborating the morphologic impression of classic clear cell RCC (Fig. 3B). Neoplastic cells in this tumor had enlarged,
FIG. 3. Histomorphology: (A) clear cell RCC, seen as nests of neoplastic cells with optically clear cytoplasm, set in a delicate vascular network. (B) Immunohistochemistry for CA-IX highlights strong membranous expression, corroborating the diagnosis of clear cell RCC. (C) High-grade B cell lymphoma, seen as discohesive neoplastic cells with moderate to scant cytoplasm and large nuclei with prominent nucleoli. (D) Immunohistochemistry for PAX-5 highlights nuclear expression, corroborating the $B$ cell phenotype of neoplastic cells. $\mathrm{RCC}=$ renal cell carcinoma; CA-IX = carbonic anhydrase-IX; PAX-5 = paired box (PAX) family.
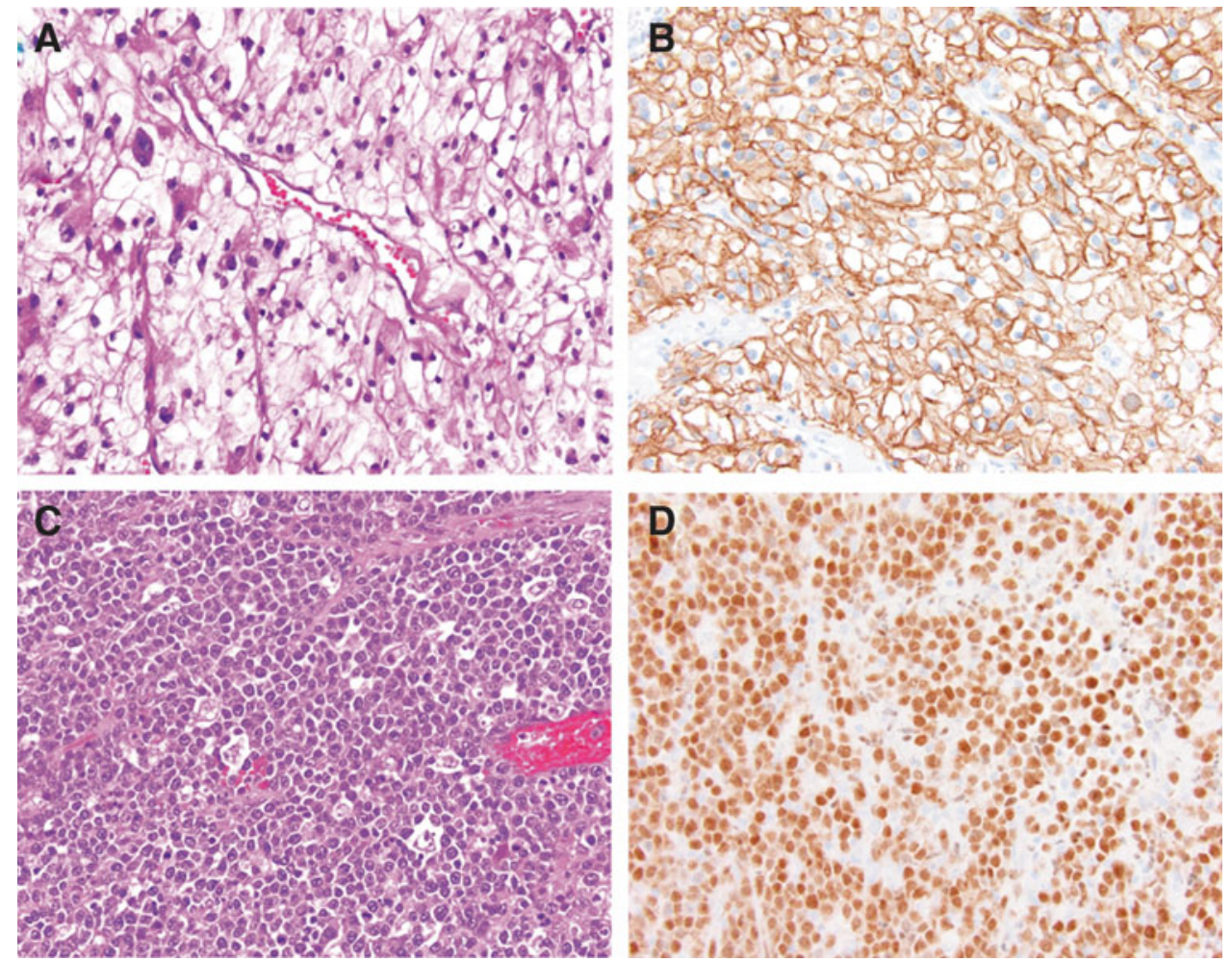
hyperchromatic nuclei, with scattered multinucleated and bizarre forms, sufficient to classify as Fuhrman (ISUP) grade 4. The larger second mass measured $8.1 \mathrm{~cm}$ and consisted of sheets of monomorphic tumor cells with moderate to scant cytoplasm and enlarged nuclei with prominent nucleoli (Fig. 3C). Immunohistochemistry on this part showed tumor cells negative for CA-IX and positive for PAX-5, CD45, MUM1, and MYC (Fig. 3D). Fluorescence in situ hybridization for $M Y C$ rearrangement was positive. The findings were interpreted as high-grade B cell lymphoma, Burkitt type, best considered an aggressive posttransplant lymphoproliferative disease (PTLD) in light of a history of organ transplantation.

The patient was evaluated by Hematology-Oncology for further management. A bone marrow biopsy was negative. Staging PET/CT scan revealed multiple new left retroperitoneal soft tissue masses. A left para-aortic lymph node core biopsy confirmed high-grade B cell lymphoma. He underwent six cycles of inpatient R-EPOCH chemotherapy complicated by an non-ST elevation myocardial infarction requiring cardiac stent placement between his fourth and fifth cycles. Restaging cross-sectional imaging performed 3 months after completing chemotherapy identified no residual disease.

\section{Discussion and Literature Review}

PRL is an extremely rare condition representing $0.7 \%$ of all extranodal tumors and $<1 \%$ of all renal lesions. ${ }^{1}$ PRL is defined as non-Hodgkin's lymphoma involving the kidney in the absence of systemic extranodal disease. To date, a total of 49 cases have been published regarding PRL. ${ }^{1}$ PRL typically presents unilaterally in males older than 40 years and it is often mistaken for RCC. The clinical presentation is nonspecific and patients may exhibit generalized symptoms, including fever, fatigue, weight loss, abdominal pain, or kidneyrelated symptoms, including a palpable flank mass, gross hematuria, thrombocytopenia, and acute kidney injury. ${ }^{2}$

The etiology of PRL is unclear as renal parenchyma does not contain lymphatic tissue. Prior reports have suggested that PRL may originate from lymph nodes within the renal sinus or lymphatic network surrounding the renal capsule. ${ }^{2}$ Chronic inflammation and repetitive injury to the parenchyma may induce lymphocyte recruitment and transformation similar to that of other extranodal mucosa-associated lymphoid tissue affecting skin and other nonlymphoid tissues. ${ }^{3}$

To our knowledge, this is the first reported case within the literature describing a collision tumor between PRL and RCC. PRL is an aggressive malignancy that may invade local tissues mimicking RCC. ${ }^{2,3}$ The patient had a history of renal transplantation and chronic immunosuppression, which placed him at a higher risk for developing malignancy, particularly PTLD. Approximately, $1 \%-5 \%$ of renal transplant recipients develop PTLD. ${ }^{4}$ Most cases of PTLD are associated with Epstein-Barr Virus (EBV) infection; however, this patient was tested EBV negative. Systemic chemotherapy with or without rituximab is considered standard of care for PRL depending on tumor grade. ${ }^{1-3}$ Although rare, it is important to consider PRL in the differential diagnosis of a solid renal mass, especially in patients with a prior history of transplantation and/or chronic immunosuppression.

\section{Conclusion}

We report a case of a collision tumor involving PRL and RCC in an immunosuppressed 62-year-old man with an incidental left renal mass on cross-sectional imaging. He underwent robot-assisted, laparoscopic left radical nephrectomy for presumed RCC without evidence for extrarenal disease. Although rare, it is important to consider PRL in the differential diagnosis of a solid renal mass, especially in patients with a prior history of transplantation and/or chronic immunosuppression.

\section{Acknowledgment}

The authors graciously thank the Penn State Health Kidney Cancer Research Fund for support of this work.

\section{Disclosure Statement}

No competing financial interests exist.

\section{References}

1. Chen X, Hu D, Fang L, et al. Primary renal lymphoma: A case report and literature review. Oncol Lett 2016;12:40014008.

2. Hagihara M, Hua J, Iwaki Y, et al. Primary renal lymphoma: A case report and literature review. Intern Med 2015;54:26552659.

3. Hart S, Ellimoottil C, Shafer D, et al. A case of primary renal lymphoma. Urology 2012;80:763-765.

4. Le J, Durand CM, Agha I, et al. Epstein-Barr virus and renal transplantation. Transplant Rev (Orlando) 2017;31:55-60.

Address correspondence to:

Jay D. Raman, MD, FACS

Division of Urology

Penn State Health

Milton S. Hershey Medical Center 500 University Drive, HO55

Hershey, PA 17033-0850

E-mail: jraman@pennstatehealth.psu.edu

Cite this article as: Kocher NJ, Leung S, Sarwani NE, Warrick JI, Raman JD (2017) Synchronous primary renal lymphoma and renal cell carcinoma: collision tumors in the same kidney, Journal of Endourology Case Reports 3:1, 87-89, DOI: 10.1089/cren.2017.0055. 